\title{
Biosensors as Implantable Medical Devices for Personalized Medicine
}

\author{
Sirinrath Sirivisoot ${ }^{1 *}$ and Thomas J. Webster ${ }^{2}$
}

${ }^{1}$ Biological Engineering, Faculty of Engineering, King Mongkut's University of Technology Thonburi, Bangkok 10140, Thailand

${ }^{2}$ School of Engineering and Department of Orthopaedics, Brown University, Providence, RI 02917, USA

\section{Editorial}

It was once thought that metal-on-metal prosthesis (orthopedic implant devices consisting of a metallic ball and socket which are used in nearly one-third of the estimated 250,000 hip replacements performed each year in the United States) were more durable than metal-on-polymer or ceramic-on-ceramic devices [1]. However, recently, analysis has demonstrated that overall $6.2 \%$ of metal-on-metal hips fail within five years, while at the same point only $1.7 \%$ of metalon-plastic hips and $2.3 \%$ of ceramic-on-ceramic hips fail [2]. This is because the wear of metal-on-metal prosthesis against each other leads to significant wear debris causing muscle and bone damage, serious infection, inflammation, and even sometimes neurological issues eventually leading to implant fracture and failure. Current problems with metal-on-metal prosthesis increase the cost of hospitalization while nations are facing the most widespread medical implant failures in decades. Therefore, it is with these problems in mind that smart biomaterials, intelligent diagnostics, and controllable drug delivery systems using biosensors have emerged leading to personalized medicine to reduce the rate of device failure and treat many health problems in real-time. Personalized medicine is an important part of the future of medicine since it has become clear that every person's immune system is unique, causing countless reactions among different patients to the same material. Only personalized medicine can address and meet the needs of such variations in everybody's immune system to ensure implant success.

Central to personalized medicine are sensors which can determine in real-time events in the body. From bench work in a medical laboratory for basic biomedical or immunological research, a biosensor has an important role in the diagnosis of any disease. The aim of researchers who are developing biosensors for personalized medicine is to quantitatively and qualitatively detect certain substances in biological liquids, bio-chemicals, and cellular events next to the implants, transforming such biological information into an understandable digital signal for human use. Sending such critical information about tissue or cellular health surrounding implants or medical devices remotely to electronic devices outside of a patient is already being realized through telemedicine and radio frequency signals. Such implantable biosensors and drug delivery technology can communicate wirelessly, much like existing peacemakers, as a personalized medicine machine.

However, where most biosensors stop is in the treatment of disease. With advances in biosensors, bioelectronics, telemetry and nanotechnology, the long-term implantable sensor chips can enable a real-time diagnosis of biological responses (bone and muscle regeneration or cell growth, proliferation and differentiation around the medical devices), implant-related complications (such as infection, inflammation or cardiovascular disease), and other common diseases (such as diabetes and cancer), and thus provide real-time treatment if it is needed. Nanotechnology (or the use of materials with length scales in the nanometer regime which mimic natural tissues) is critical to the field of implantable biosensors as there is now a growing body of evidence that nanomaterials can prolong drug delivery, increase tissue growth, decrease inflammation, and inhibit infection; all criteria critical for the long-term functioning of biosensors in the body. Given the opportunity to explore the causes of biological events (such as bone in growth) or diseases (such as infection) faster with an implantable biosensor, personalized medicine can provide more timely treatment and appropriate remedies to increase life expectancy after medical device procedures to improve global healthcare. A long-term implantable sensor detecting biochemical and cellular responses in an individual with controlled drug delivery, can function together truly as personalized medicine, where health abnormalities can be detected and fixed immediately; we are on the cusp of such advances, perhaps even in the next decade.

\section{References}

1. http://www.nytimes.com/

2. http://www.bbc.co.uk/news/health-17337993
*Corresponding author: Sirinrath Sirivisoot, Biological Engineering, Faculty of Engineering, King Mongkut's University of Technology Thonburi, Bangkok 10140, Thailand Tel: +(66) 083-014-1716; E-mail: Sirinrath@gmail.com, Sirinrath.Sir@kmutt.ac.th

Received March 19, 2012; Accepted March 20, 2012; Published March 22, 2012

Citation: Sirivisoot S, Webster TJ (2012) Biosensors as Implantable Medical Devices for Personalized Medicine. J Biosens Bioelectron 3:e104. doi:10.4172/2155-6210.1000e104

Copyright: (c) 2012 Sirivisoot S, et al. This is an open-access article distributed under the terms of the Creative Commons Attribution License, which permits unrestricted use, distribution, and reproduction in any medium, provided the original author and source are credited. 